Preference for different routes of administration varied. Ketamine is initiated in outpatient clinics/ day units, and in patients' homes. IV ketamine is only prescribed in hospital/hospice. $86 \%$ of consultants discharge patients on ketamine into the community. An estimated 255 patients received ketamine in the previous 12 months. Concomitant opioid reduction varied greatly between physicians. 12/15 physicians switch ketamine routes from SC to PO during treatment. PO and SC ketamine are being used indefinitely. IV treatment is only used as 'burst' treatment. $87 \%$ give PRN ketamine. Test dose administration varies greatly $(53 \%$ SC and $100 \%$ IV). 5/15 wanted training on IV ketamine. Barriers to ketamine use include cost, community support, and lack of IV pumps. 93\% do not use a standardised recording tool. Most organisations do not have a written protocol.

Conclusion There is significant variation in practice of ketamine use. Despite recent adverse evidence, SC ketamine is most commonly prescribed. Many patients are treated indefinitely and are at risk of side effects. Further studies are needed to establish national usage and to identify priorities for research.

\section{P 155 KETAMINE USE IN PALLIATIVE CARE WITHIN THE NORTH TRENT CANCER NETWORK (NTCN)}

Samuel Fingas, ${ }^{1}$ Ashique Ahamed, ${ }^{3}$ Sam H Ahmedzai ${ }^{3,1}$. ${ }^{1}$ Sheffield Teaching Hospitals, Sheffield, United Kingdom; ${ }^{2}$ University of Sheffield; ${ }^{3}$ Central Manchester University Hospitals, Manchester, United Kingdom

10.1136/bmjspcare-2014-000654.196

Background Ketamine is a potent analgesic with potentially serious adverse effects (eg dysphoria, bladder dysfunction), being used in palliative medicine. Its evidence base is limited and a recent RCT using the SC route in cancer pain failed to show efficacy. Neither route nor dosing are standardised, which raises questions for clinical governance in palliative care services.

Aims To characterise ketamine use in palliative medicine within NTCN, with specific reference to variations in route and dosing. Method A short online questionnaire was developed using SurveyMonkey, to capture NTCN palliative medicine consultants' current practices, preferences and opinions. Reminders were used to ensure compliance.

Results Response rate 15/15 (100\%). All had prescribed ketamine in their current post: $100 \%$ via SC route, $93 \%$ oral, $27 \%$ IV. 